Molecules 2003, 8, 418-429

molecules

ISSN 1420-3049

http://www.mdpi.org/

\title{
Theoretical Study on the Interaction of o-Tetrafluorophenylene Mercury with Ethylene and Acetylene ${ }^{\dagger}$
}

\author{
Eduardo A. Castro*
}

CEQUINOR, Departamento de Química, Facultad de Ciencias Exactas, Universidad Nacional de La Plata, Calles 47 y 115, C.C. 962, La Plata 1900, Argentina

$\dagger \quad$ Previously published on the ChemWeb Chemistry Preprint Server as CPS: physchem/0207010

* To whom correspondence should be addressed; e-mail: castro@dalton.quimica.unlp.edu.ar / jubert@arnet.com.ar

Received: 4 February 2003; in revised form: 17 March 2003 / Accepted: 20 March 2003 / Published: 31 May 2003

\begin{abstract}
The results of a theoretical study on the interaction of $o$-tetrafluorophenylene mercury with ethylene and acetylene are reported. The AM1 molecular orbital semiempirical method is applied through a complete optimization procedure without any restrictions to find the optimal equilibrium geometries. The comparative capabilities of ethylene and acetylene molecules to form stable complexes with $o$-tetrafluorophenylene mercury are analyzed and their corresponding bonding features are discussed. Ethylene seems to be capable of forming a molecular complex while acetylene is predicted to be incapable of doing so.
\end{abstract}

Keywords: $\mu_{6}-\eta^{2}: \eta^{2}: \eta^{2}: \eta^{2}: \eta^{2}: \eta^{2}$ bonding mode - o-tetrafluorophenylene mercury ethylene - acetylene - AM1 semiempirical method. 


\section{Introduction}

The affinity of mercury for aromatic compounds is well documented. While electrophilic mercuration reactions [1] and $\pi$-complex formation [2,3] substantiate the high affinity of $\mathrm{Hg}^{+2}$ cations for arenes, weaker but measurable interactions also occur between arenes and organomercurials [4]. Recently, it has been reported that benzene has been sandwiched between two identical planar trimetallic molecules (o-tetrafluorophenylene mercury, $\mathbf{1}$, Scheme 1 ) in such a way that each of the six metal atoms coordinates to a different $\mathrm{C}-\mathrm{C}$ bond of the benzene [5].

\section{Scheme 1}

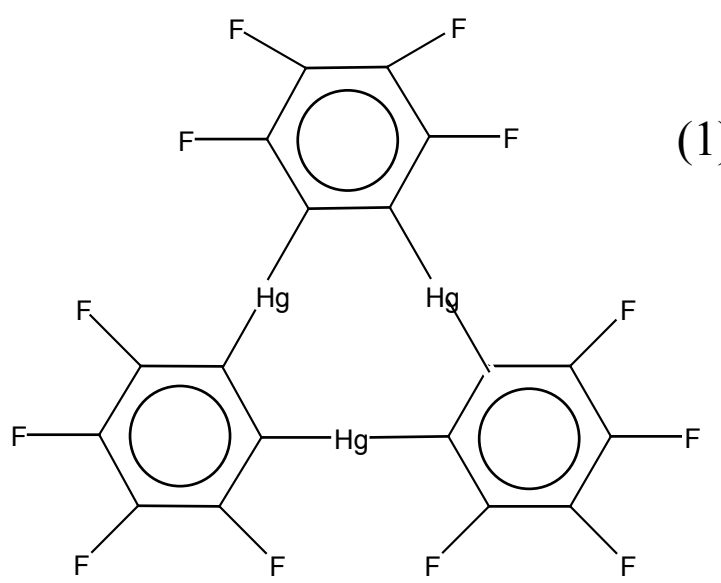

In fact, the reaction of benzene with trimeric $o$-tetrafluorophenylene mercury leads to the formation of a supramolecule that contains sandwiched $\mu_{6}-\eta^{2}: \eta^{2}: \eta^{2}: \eta^{2}: \eta^{2}: \eta^{2}$ benzene. The interaction represents a new bonding mode for benzene and it represents a novel possibility of obtaining complexes in which this aromatic molecule is coordinated to more than three metals when two adequately chosen polydentate Lewis acid molecules interact concomitantly with a unique benzene molecule. Authors concluded from single-crystal X-ray analysis of the complex that extended stacks of $1 \cdot \mathrm{C}_{6} \mathrm{H}_{6}$ run parallel to one another.

More recently the present author has performed two theoretical studies of this new complex using Molecular Mechanics techniques and the AM1 semiempirical method in order to complement previous experimental findings [6,7]. These calculations allow one to know which are main factors governing the different chemical bonds and besides, to predict the possible formation of new similar compounds. This last point is particularly important because Tsunoda and Gabbai [5] reported that they were investigating the interaction of $\mathbf{1}$ with acetylene experimentally, although up to the present moment they have not published any paper in this regard, as far as this author is aware. Thus, I have considered it interesting to carry out a theoretical study at the AM1 semiempirical level on the interaction of 1 with ethylene and acetylene in order to assess the molecular stabilities of these new complexes. 


\section{Results and Discussion}

The main structural data for isolated ethylene, acetylene and 1, derived from the AM1 method, are given in Table 1, where distances are in $\AA$ and angles in degrees. When more than three values for a given structural parameter are obtained from the calculations, the extreme (i.e. minimum and maximum) values are indicated between brackets.

Table 1. Main structural data derived from the AM1 study for $\mathbf{1}$ and $\mathbf{X}$ molecules

\begin{tabular}{|l|c|c|c|}
\hline $\begin{array}{l}\text { Geometrical } \\
\text { Parameter }\end{array}$ & $\mathbf{1}$ & Ethylene & Acetylene \\
\hline $\boldsymbol{d}(\boldsymbol{C}-\boldsymbol{H g})$ & 2.074 & - & - \\
$\boldsymbol{d}(\boldsymbol{C}-\boldsymbol{F})$ & $1.350 ; 1.358$ & - & - \\
$\boldsymbol{d}(\boldsymbol{C}-\boldsymbol{C})$ & {$[1.389 ; 1.419]$} & 1.326 & 1.195 \\
$\boldsymbol{d}(\boldsymbol{C}-\boldsymbol{H})$ & - & 1.098 & 1.061 \\
$\boldsymbol{C}-\boldsymbol{C}-\boldsymbol{C}$ - angle & $119.2 ; 120.0 ; 120.8$ & - & - \\
$\boldsymbol{C}-\boldsymbol{C}-\boldsymbol{H}$ angle & - & 122.8 & 180 \\
$\boldsymbol{C}-\boldsymbol{H} \boldsymbol{-}-\boldsymbol{C}$ angle & $148.6 ; 158.8$ & - & - \\
$\boldsymbol{C}-\boldsymbol{C}-\boldsymbol{F}$ angle & $113.9 ; 123.0$ & - & - \\
\hline
\end{tabular}

The global structural data for $\mathbf{1} \cdot \mathbf{X}$ and $\mathbf{1} \cdot \mathbf{X} \cdot \mathbf{1}$ are given in Tables 2, 3 and 4 . The second complexes have been computed for both the staggered and eclipsed conformations. Molecular structures at the equilibrium geometry for these complexes are presented in Figures 1-6.

Table 2. Significant structural data for $\mathbf{1} \cdot \mathbf{X}^{*}$

\begin{tabular}{|c|c|c|c|c|}
\hline \multirow{2}{*}{$\begin{array}{l}\text { Geometrical } \\
\text { parameter }\end{array}$} & \multicolumn{2}{|c|}{$X=$ ethylene } & \multicolumn{2}{|c|}{$X=$ acetylene } \\
\hline & Ethylene & 1 & Acetylene & 1 \\
\hline$d(C-H g)$ & - & {$[2.046 ; 2.123]$} & - & {$[2.048 ; 2.119]$} \\
\hline$d(C-F)$ & - & {$[1.349 ; 1.389]$} & - & {$[1.349 ; 1.388]$} \\
\hline$d(C-C)$ & 1.343 & {$[1.383 ; 1.423]$} & 1.209 & {$[1.383 ; 1.423]$} \\
\hline$d(C-H)$ & $1.105 ; 1.107$ & - & $1.071 ; 1.072$ & - \\
\hline$C-C-C$ angle & - & {$[116.9 ; 123.4]$} & - & {$[116.9 ;, 123.3]$} \\
\hline$C-C-H$ angle & $122.3 ; 122.6 ; 122.7$ & - & $174.0 ; 176.7$ & - \\
\hline $\mathrm{C}$-Hg-C angle & - & $163.9 ; 166.3 ; 141.3$ & - & $145.3 ; 165.9 ; 166.6$ \\
\hline$C-C-F$ angle & - & {$[113.9 ; 123.1]$} & - & {$[113.9 ; 123.0]$} \\
\hline
\end{tabular}

* Data expressed as in Table 1 
Table 3. Significant structural data for $\mathbf{1} \cdot$ ethylene $\mathbf{1}$ *

\begin{tabular}{|c|c|c|c|c|}
\hline \multirow{2}{*}{$\begin{array}{l}\text { Geometrical } \\
\text { Parameter }\end{array}$} & \multicolumn{2}{|c|}{ Staggered } & \multicolumn{2}{|c|}{ Eclipsed } \\
\hline & Ethylene & 1 & Ethylene & 1 \\
\hline$d(C-H g)$ & - & {$[2.118 ; 2.262]$} & - & $2.050 ; 2.096$ \\
\hline$d(C-F)$ & - & {$[1.348 ; 1.390]$} & - & {$[1.348 ; 1.388]$} \\
\hline$d(C-C)$ & 1.670 & {$[1.397 ; 1.431]$} & 1.326 & {$[1.383 ; 1.423]$} \\
\hline$d(C-H)$ & $0.680 ; 0.722 ; 0.805$ & - & $1.098 ; 1.099$ & - \\
\hline$C-C-C$ angle & - & {$[117.1 ; 123.9]$} & - & {$[117.1 ; 123.1]$} \\
\hline$C-C-H$ angle & {$[116.5 ; 134.0]$} & - & 122.7 & - \\
\hline $\mathrm{C}-\mathrm{Hg}-\mathrm{C}$ angle & - & {$[125.2 ; 131.9]$} & - & $167.2 ; 167.3$ \\
\hline$C-C-F$ angle & - & {$[114.6 ; 122.5]$} & - & {$[113.9 ; 123.0]$} \\
\hline
\end{tabular}

* Data expressed as in Table 1

Table 4. Significant structural data for 1 acetylene 1 *

\begin{tabular}{|c|c|c|c|c|}
\hline \multirow{2}{*}{$\begin{array}{l}\text { Geometrical } \\
\text { Parameter }\end{array}$} & \multicolumn{2}{|c|}{ Staggered } & \multicolumn{2}{|c|}{ Eclipsed } \\
\hline & Ethylene & 1 & Ethylene & 1 \\
\hline$d(C-H g)$ & - & {$[2.045 ; 2.114]$} & - & {$[2.047 ; 2.126]$} \\
\hline$d(C-F)$ & - & {$[1.348 ; 1.388]$} & - & {$[1.348 ; 1.388]$} \\
\hline$d(C-C)$ & 1.207 & {$[1.382 ; 1.425]$} & 1.207 & {$[1.383 ; 1.424]$} \\
\hline$d(C-H)$ & $1.071 ; 1.072$ & - & $1.071 ; 1.072$ & - \\
\hline$C-C-C$ angle & - & {$[117.0 ; 123.2]$} & - & {$[117.1 ; 123.4]$} \\
\hline C-C-H angle & $175.6 ; 176.4$ & - & $175.6 ; 176.4$ & - \\
\hline $\mathrm{C}-\mathrm{Hg}$-C angle & - & {$[151.6 ; 178.3]$} & - & {$[142.1 ; 178.4]$} \\
\hline$C-C-F$ angle & - & {$[113.7 ; 123.3]$} & - & {$[113.9 ; 123.0]$} \\
\hline
\end{tabular}

* Data expressed as in Table 1 
Molecules 2003, 8

Figure 1

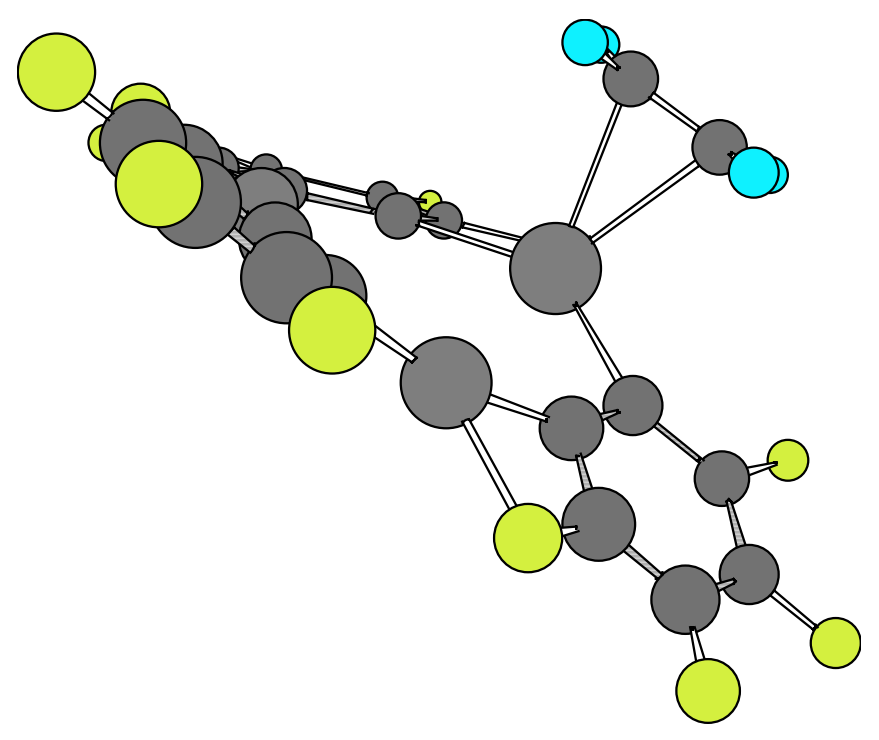

Figure 2

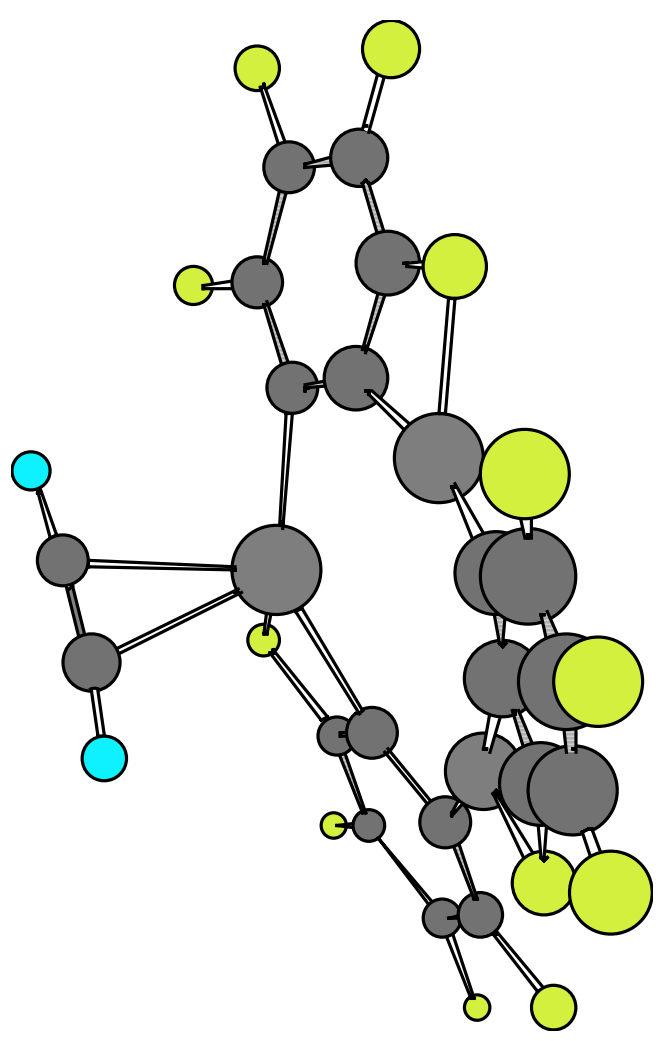


Molecules 2003, 8

Figure 3

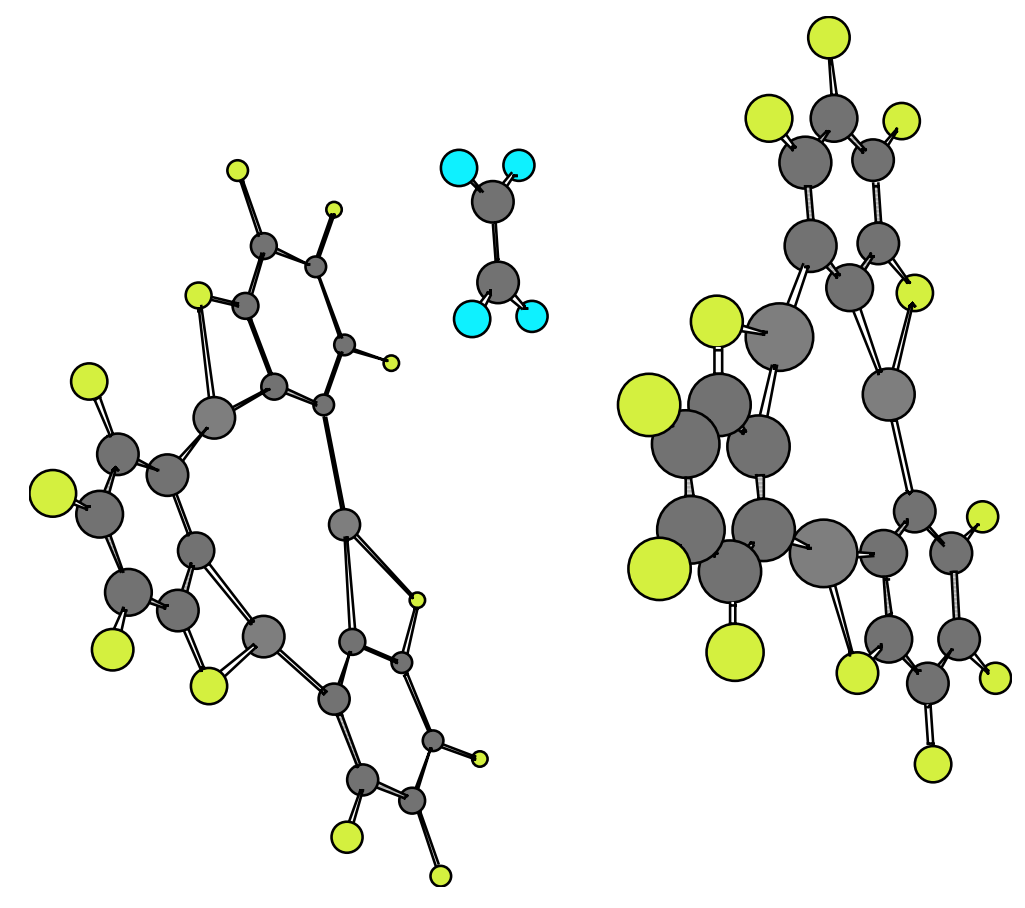

Figure 4

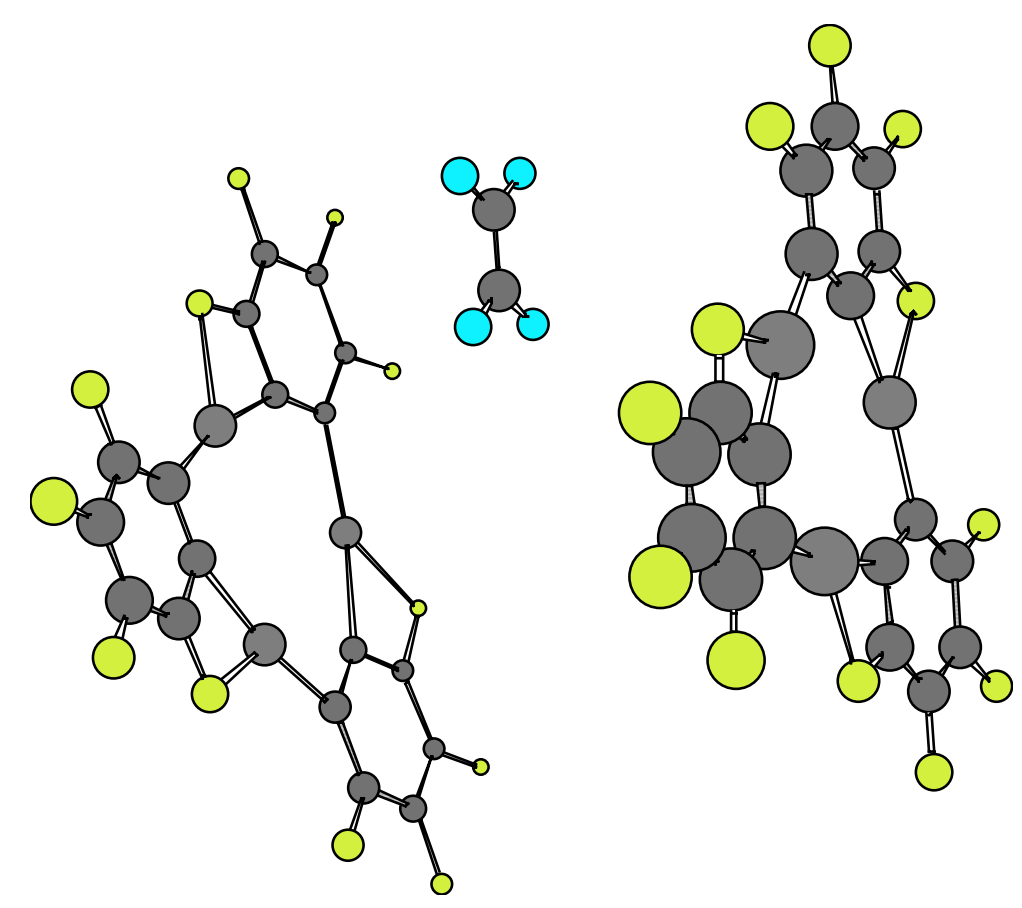


Molecules 2003, 8

Figure 5

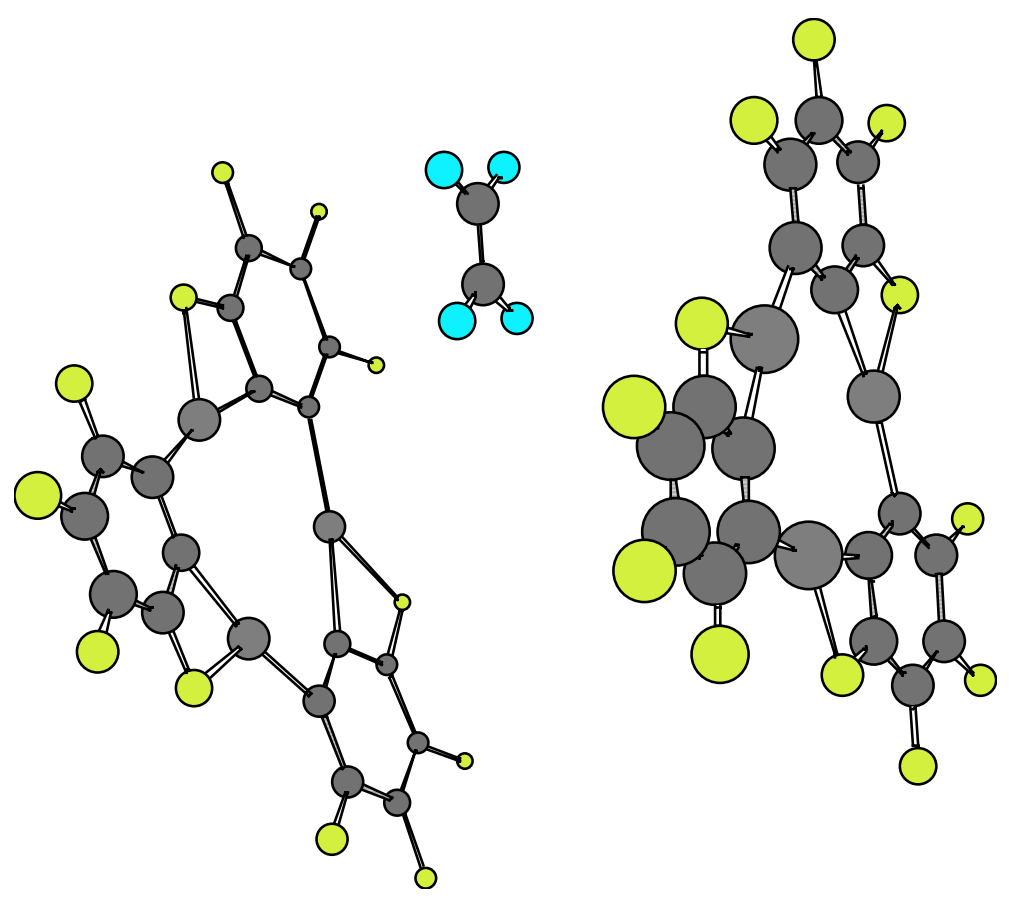

Figure 6

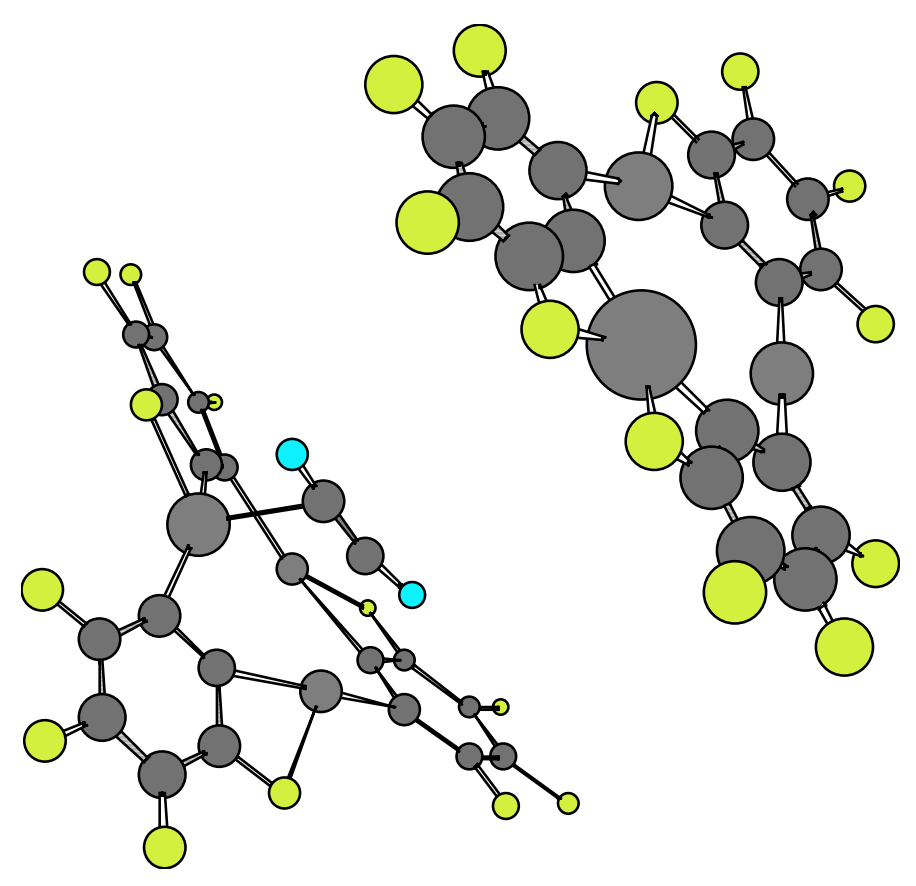


In order to get an approximate measurement of the complex stability we have also calculated the stability energy, defined as

$$
\mathrm{E}_{\mathrm{cs}}=\mathrm{E}_{\text {complex }}-\mathrm{E}_{\mathrm{X}}-\mathrm{E}_{1}
$$

for $\mathbf{1} \cdot \mathbf{X}$ complexes

and

$$
\mathrm{E}_{\mathrm{sc}}=\mathrm{E}_{\text {complex }}-\mathrm{E}_{\mathrm{X}}-\mathrm{E}_{\mathbf{1} \cdot \mathbf{1}}
$$

for $\mathbf{1} \cdot \mathbf{X} \cdot \mathbf{1}$ complexes, and results are given in Table 5 .

Table 5. Complex stability for different molecular complexes (kcal/mol).

\begin{tabular}{|l|c|}
\hline \multicolumn{1}{|c|}{ Complex } & $\boldsymbol{E}_{\boldsymbol{s c}}{ }^{*}$ \\
\hline $\mathbf{1} \cdot$ ethylene & -38.63 \\
$\mathbf{1} \cdot$ acetylene & -40.63 \\
$\mathbf{1} \cdot$ ethylene$\cdot \mathbf{1}$ (eclipsed) & 0.53 \\
$\mathbf{1} \cdot$ ethylene $\mathbf{1}$ (staggered) & -40.65 \\
$\mathbf{1} \cdot$ acetylene·1 (eclipsed) & -0.09 \\
$\mathbf{1} \cdot$ acetylene $\mathbf{1}$ (staggered) & 0.00 \\
$\mathbf{1} \cdot \mathbf{1}$ (eclipsed) & -63.62 \\
$\mathbf{1} \cdot \mathbf{1}$ (staggered) & -108.14 \\
\hline
\end{tabular}

* See definitions (5) and (6) in the text.

The comparison of main structural data derived from AM1 for isolated $\mathbf{1}$ and $\mathbf{X}(\mathrm{X}=$ ethylene, acetylene) molecules and the $\mathbf{1} \cdot \mathbf{X}$ adducts shows there exists an effective molecular interactions in such a way as to yield significant changes in equilibrium bond lengths and bond angles. In fact, when looking at Figures 1 and 2, respectively, it may be seen that there is a chemical bonding between $\mathrm{X}$ and one of the $\mathrm{Hg}$ atoms of the $o$-tetrafluorophenylene mercury molecule. Besides, the calculation of the $\mathrm{E}_{\mathrm{sc}}$ for these complexes shows a net stabilizing effect, as can be verified from results presented in Table 5. The $\mathrm{E}_{\mathrm{sc}}$ values for both complexes (i.e. $-38.63 \mathrm{kcal} / \mathrm{mol}$ and $-40.63 \mathrm{kcal} / \mathrm{mol}$, respectively) are rather similar. It is interesting to point out that $\mathbf{X}$ locates at a geometrical position so as to have a maximum interaction with a given $\mathrm{Hg}$ atom instead of positioning at a more symmetrical and equidistant place regarding the three $\mathrm{Hg}$ atoms.

The geometrical changes taking place when $\mathbf{1} \cdot \mathbf{X}$ complexes form are particularly noticeable. In fact, $\mathrm{C}-\mathrm{C}$ and $\mathrm{C}-\mathrm{H}$ bond distances for $\mathbf{X}$ increase and the same happens with the $\mathrm{C}-\mathrm{C}-\mathrm{H}$ angles. Besides, a slight molecular deformation also happens in such a way that in the complexes there are 
several geometrical equilibrium bond and angle parameters instead of just one (i.e. more than one $\mathrm{C}-\mathrm{H}$ distance and one C-C-H angle). A similar feature is produced for $\mathbf{1}$, in such a way that calculations predict, for example, a relatively varied set of numerical data for each geometrical parameter corresponding to the equilibrium conformation, as shown in Table 2.

The analysis of the $\mathbf{1} \cdot \mathbf{X} \cdot \mathbf{1}$ complexes has been performed at the relative eclipsed and staggered locations of both $\mathbf{1}$ molecules. The $\mathrm{E}_{\mathrm{sc}}$ for the dimers $\mathbf{1} \cdot \mathbf{1}$ at the eclipsed and staggered configurations are $-63.62 \mathrm{kcal} / \mathrm{mol}$ and $-108.14 \mathrm{kcal} / \mathrm{mol}$, respectively. Equilibrium conformations are shown in Figures 7 and 8 .

Figure 7

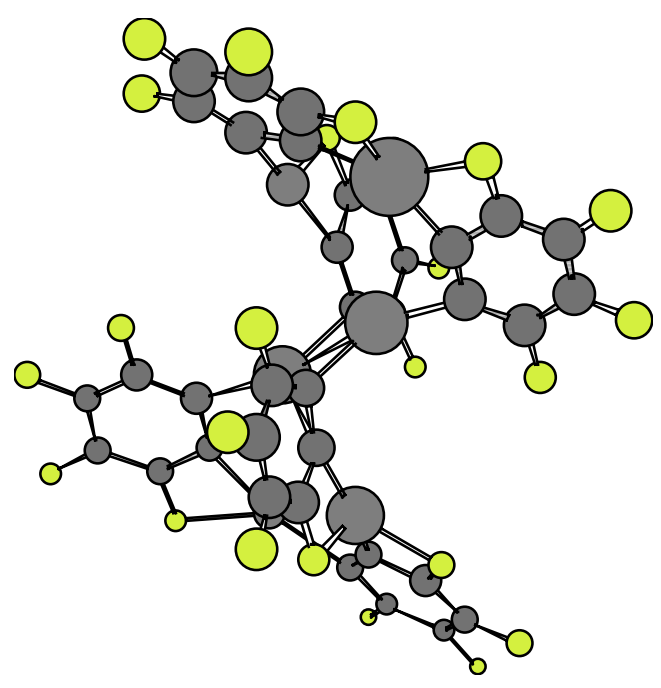

Figure 8

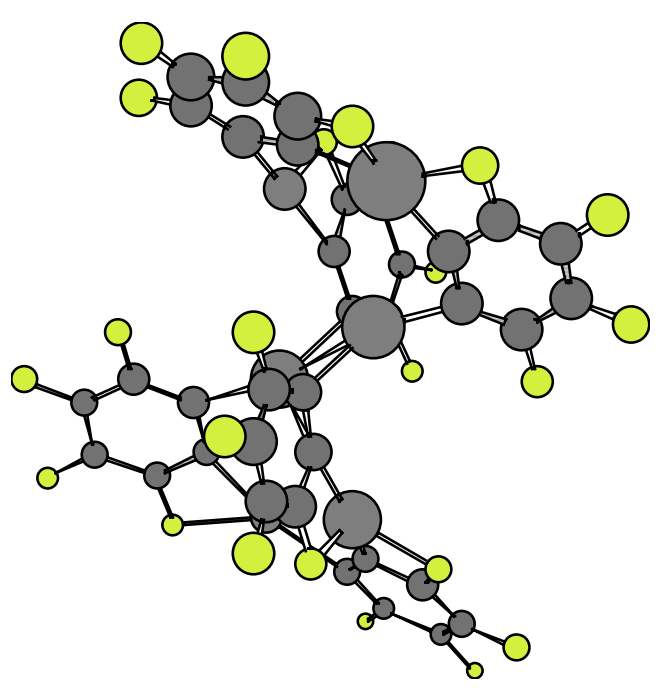


The greater stability of the staggered conformation at the equilibrium point with respect to the eclipsed one is clearly understood on the basis of the stronger atomic repulsion of equivalent atoms in each monomer 1.

The comparison of equilibrium geometries of the isolated molecules with regards to those found at the complexes $\mathbf{1} \cdot \mathbf{X} \cdot \mathbf{1}$ once again shows considerable distortions, which are larger than those found in the $\mathbf{1} \cdot \mathbf{X}$ complexes. The variations in numerical values for a given molecular structure parameter are also rather ample, which implies remarkable distortions in the equilibrium with respect to the more symmetrical features of the isolated species (see Tables 1, 3 and 4). These properties can be seen depicted in Figures 3-6. However, when considering the energetic stability one finds that only the $\mathbf{1} \cdot$ ethylene $\mathbf{1}$ complex in the staggered conformation presents a noticeable $\mathrm{E}_{\mathrm{sc}}$ negative value $(-40.65$ $\mathrm{kcal} / \mathrm{mol}$, see Table 5). Although this stability energy value is about $40 \%$ less than that corresponding to the dimer $1 \cdot 1$ (i.e. $-108.14 \mathrm{kcal} / \mathrm{mol}$ ), it is enough to confer a possible molecular existence to the not-yet synthesized complex. The $\mathrm{E}_{\mathrm{sc}}$ values for the other 1.X.1 complexes are practically zero, so that semiempirical AM1 predicts they cannot exist as such. The analysis of main occupied molecular orbitals shows a donating effect from the $\pi$-MO's of $\mathbf{X}$ towards available $6 \mathrm{p}$ orbitals of mercury. This feature is similar to that one postulated for the $\mathbf{1} \cdot$ benzene $\mathbf{1}$ complex discussed previously $[5,6]$.

The main difference between present results for $\mathbf{1} . \mathbf{X} \cdot \mathbf{1}$ complexes and the experimental evidence for $1 \cdot$ benzene 1 complex is that in the former case, a significant distortion in $\mathbf{1}$ and $\mathbf{X}$ with respect to the isolated species takes place when the adduct is formed, while in the latter case, only minute distortions happen. In particular, molecules of $\mathbf{1}$ deviate to a large extent from planarity and each stack consists of staggered molecules of $\mathbf{1}$ that sandwich $\mathbf{X}$ molecules.

\section{Conclusions}

A complete geometrical optimization for $\mathbf{1} \cdot \mathbf{X}$ and $\mathbf{1} \cdot \mathbf{X} \cdot \mathbf{1}$ complexes was performed at the semiempirical AM1 level. The optimization procedure was carried out without any restriction at all. Theoretical predictions reveal the possibility of formation of $\mathbf{1} \cdot \mathbf{e t h y l e n e} \mathbf{1}$ complex at the staggered conformation, while other complexes have a zero energy of molecular stability. This study makes up a theoretical answer to the possible formation of $\mathbf{1} \cdot \mathbf{X} \cdot \mathbf{1}$ complexes when $\mathbf{X}=$ ethylene, acetylene, which was raised recently by Tsunoda and Gabbai [5] after the report that the reaction of benzene with trimeric $o$-tetrafluorophenylene mercury leads to the formation of a supramolecule that contains sandwiched $\mu_{6}-\eta^{2}: \eta^{2}: \eta^{2}: \eta^{2}: \eta^{2}: \eta^{2}$-benzene.

\section{Methods}

The key components of the Roothaan-Hall equations for a closed shell system are

$$
\mathbf{F C}=\mathbf{S C E}
$$




$$
\begin{aligned}
& \mathrm{F}_{\mu \nu}=\mathrm{H}_{\mu \nu}^{\text {core }}+\sum_{\lambda=1}^{\mathrm{K}} \sum_{\sigma=1}^{\mathrm{K}} \mathrm{P}_{\lambda \sigma}[(\mu \nu \mid \lambda \sigma)-(\mu \lambda \mid v \sigma) / 2] \\
& \mathrm{P}_{\lambda \sigma}=2 \sum_{\mathrm{i}=1}^{\mathrm{N} / 2} \mathrm{c}_{\lambda_{\mathrm{i}}} \mathrm{c}_{\sigma \mathrm{i}} \\
& \mathrm{H}_{\mu \nu}^{\text {core }}=\int \mathrm{dv}_{1} \varphi_{\mu}(1)\left[-1 / 2 \nabla^{2}-\sum_{\mathrm{A}=1}^{\mathrm{M}} \mathrm{Z}_{\mathrm{A}} / \mathbf{r}_{1}-\mathrm{R}_{\mathrm{A}} \mid\right] \varphi_{\nu}(1)
\end{aligned}
$$

In ab initio calculations all computations of the Fock matrix $\mathrm{F}_{\mu \nu}$ are calculated using equation (2), irrespective of whether the basis functions $\varphi_{\mu}, \varphi_{v}, \varphi_{\lambda}$ and $\varphi_{\sigma}$ are on the same atom, on atoms that are bonded or on atoms that are not formally bonded. The greatest proportion of the time required to perform an $a b$ initio Hartree-Fock SCF calculation is invariably spent calculating and manipulating integrals. The most obvious way to reduce the computational effort is therefore to neglect or approximate some of these integrals. Semi-empirical methods achieve this in part by explicitly considering only the valence electrons of the system; the core electrons are subsumed into the nuclear core. The rationale behind this approximation is that the electrons involved in chemical bonding and other phenomena that we might wish to investigate are those in the valence shell. The semiempirical calculations invariably use basis sets comprising Slater-type $s, p$ and sometimes $d$ orbitals. The orthogonality of such orbitals enables further simplifications to be made to the equations.

The Austin Model 1 (AM1) model was produced by Dewar's group [8] and it was designed to eliminate the problems with MNDO which were considered to arise from a tendency to overestimate repulsions between atoms separated by distances approximately equal to the sum of their van der Waals radii [9]. The strategy adopted was to modify the core-core term using Gaussian functions. Both attractive and repulsive Gaussian functions were used; the attractive Gaussian were designed to overcome the repulsion directly and were centered in the region where the repulsions were too large. Repulsive Gaussian functions were centered at smaller internuclear separations. Overall, AM1 was a significant improvement over MNDO and many of the deficiencies associated with the core repulsion were corrected, but at the same time it has some drawbacks [10].

The calculations were performed at the AM1 semi-empirical level, using the standard HYPERCHEM $^{\circledR}$ package [11]. The Polak-Ribiére first-order minimization algorithm was chosen for present calculations, which is frequently employed in molecular modeling. This method gradually changes the coordinates of the atoms as they move closer and closer to the minimum point. The starting point for each interaction $(\mathrm{k})$ is the configuration obtained from the previous step which is represented by the multidimensional position vector $\mathbf{x}_{\mathrm{k}-1}$. For the first interaction the starting point is the initial configuration of the system provided by the user (i.e. the vector $\mathbf{x}_{1}$ ) through, for example, the Model Building option included in the computational program. 
The conjugate gradient both the gradients and the direction of successive steps are orthogonal but the directions are conjugate (indeed, the method is more properly called the conjugate directions method). A set of conjugate directions has the property that for a quadratic function of $\mathrm{M}$ variables, the minimum will be reached in M steps. The root mean square (RMS) gradient was chosen equal to 0.01 $\mathrm{kcal} / \mathrm{mol}$.

The calculations to obtain a total molecular optimization without any restriction were performed for the isolated title molecules and complexes $\mathbf{1} \cdot \mathbf{X}(\mathbf{X}=$ ethylene, acetylene $)$ and $\mathbf{1} \cdot \mathbf{X} \cdot \mathbf{1}$ in the eclipsed and staggered conformations. Every computational run was completed within a short time on a Pentium III PC equipped with a $1 \mathrm{GHz}$ CPU and 256 Mbytes of RAM.

Complete geometrical parameter results for every molecule and complex in their equilibrium conformations mentioned in this paper as well as main molecular orbital surfaces of bonding orbitals are available upon request to the author.

\section{References}

1. Lau, W.; Kochi, J. K. J. Am. Chem. Soc. 1986, 108, 6720.

2. Lau, W.; Huffman, J. C.; Kochi, J. K. J. Am. Chem. Soc. 1982, 104, 5515.

3. Mamude, L. C.; Dean, P. A. W.; Sefcik, M. D.; Schaefer, J. J. Organomet. Chem. 1982, 226, 104.

4. Kuz'mina, L. G.; Struchkov, Yu. T. Croat. Chem. Acta, 1984, 57, 701 and references therein.

5. Tsunoda, M.; Gabbai, F. P. J. Am. Chem. Soc. 2000, 122, 8335.

6. Castro, E. A. J. Mol. Struct. THEOCHEM 2002, 619, 45.

7. Castro, E. A. J. Struct. Chem. (in press).

8. Dewar, M. J. S.; Zoeblisch, E. G.; Healy, E. F.; Stewart, J. J. P. J. Am. Chem. Soc. 1985, 107, 3902.

9. Leach, R. Molecular Modelling. Principles and Applications; Prentice Hall: Harlow (U.K.), 1996; pp.102-103.

10. Hehre, W. J.; Shusterman, A. J.; Wayne Huang, W. A Laboratory Book of Computational Organic Chemistry; Wavefunction, Inc.: CA, 1996; p. 5.

11. HYPERCHEM ${ }^{\circledR}$ for Windows, Hypercube, Inc., Florida (USA), 1994.

Sample availability: Not applicable

(C) 2003 by MDPI (http://www.mdpi.org). Reproduction is permitted for noncommercial purposes. 